\title{
The angular momentum of vectorial non-paraxial fields and the role of radial charges in orbit-spin coupling
}

\author{
Omar El Gawhary ${ }^{1,2, *}$ and Paul Urbach $^{2}$ \\ ${ }^{1}$ VSL Dutch Metrology Institute, Thijsseweg 112629 JA Delft, Netherlands \\ ${ }^{2}$ Optics Research Group, Delft University of Technology, Lorentzweg 12628 CJ Delft, Netherlands
}

\begin{abstract}
Electromagnetic fields carry a linear and an angular momentum, the first being responsible for the existence of the radiation pressure and the second for the transfer of torque from electromagnetic radiation to matter. The angular momentum is considered to have two components, one due to the polarization state of the field, usually called Spin Angular Momentum (SAM), and one due to existence of topological azimuthal charges in the field phase profile, which leads to the Orbital Angular Momentum (OAM). For non-paraxial fields these two contributions are not independent of each other, something which is described as spin-orbit coupling. It has been recently proved that electromagnetic fields necessarily carry also invariant radial charges that, as discussed in this work, play a key role in the angular momentum. Here we show that the total angular momentum consists in fact of three components: one component only dependent on the spin of the field, another dependent on the azimuthal charges carried by the field and a third component dependent on the spin and the radial charges contained in the field. By properly controlling the number and coupling among these radial charges it is possible to design electromagnetic fields with a desired total angular momentum. In this way it is also possible to discover fields with no orbital angular momentum and a spin angular momentum typical of spin-3/2 objects, irrespective of the fact that photons are spin-1 particles.
\end{abstract}

\section{Introduction}

Electromagnetic fields carry an angular momentum which is responsible for the transfer of torque from light to matter. After the work by Allen et. al [1] the interest on the angular momentum of light, its origin and effect on matter, has gradually grown through the years and currently represents an active area of investigation, especially in view of possible applications to high-capacity optical communications, novel metrology techniques, classical and quantum imaging [2-5]. There are essentially two sources the angular momentum: one due to the polarization state of the field (spin angular momentum or SAM, for short) and the other due to the presence of a helical wavefront in the electromagnetic field (orbital angular momentum or OAM)[6-15]. In this work we discuss an insightful expression of the total angular momentum (TAM) in terms of the $s$ and $p$ components of the angular spectra of the field. We then use that expression of the TAM and combine it with the recently introduced representation of a field in terms of Helmholtz Natural Modes which allows us to identify two types of topological

\footnotetext{
*e-mail: o.elgawhary@tudelft.nl
} 
charges carried by a field: an azimuthal charge and a radial charge. The existence of azimuthal charges has been known for long as they are linked to the orbital angular momentum of a field. On the other hand, the existence of topological radial charges seemed to have remained unnoticed to the scientific community. Interestingly, as we are going to present in this work, radial topological charges are responsible for the orbit-spin coupling in non-paraxial fields. Once the main mechanism behind the generation of the Total Angular Momentum (TAM) of a field is clear, we can show the existence of fields endowed with only a SAM contribution, which however corresponds to spin-3/2 particles, something surprising when dealing with electromagnetic fields. In this work we will consider the time-averaged TAM, which can be written in the following way

$$
\boldsymbol{J}=\frac{\varepsilon_{0} \mu_{0}}{2} \operatorname{Re}\left\{\int\left[\mathbf{r} \times\left(\mathbf{E} \times \mathbf{H}^{\star}\right)\right] d \mathbf{r}\right\}
$$

where the $\star$ symbol stands for complex conjugation. We will show that for a beam propagating along the $z$-direction, the $\mathrm{z}$-component of the TAM per unit length can be expressed in Fourier space as

$$
\mathfrak{J}_{z}=\operatorname{Re}\left\{\frac{\varepsilon_{0}}{2 i \omega} \int\left[\left(A_{s}^{\star} \frac{\partial A_{s}}{\partial \varphi}+A_{p}^{\star} \frac{\partial A_{p}}{\partial \varphi}\right)-\frac{(2 \pi)^{2}}{k} \frac{\rho^{2}}{k_{z}} A_{s}^{\star} A_{p}\right] \rho d \rho d \varphi\right\}
$$

Eq.2 shows that TAM can in fact be expressed in terms of the sum of a s-polarized contribution (which we will call S-TAM), a p-polarized contribution (which we will denote as P-TAM) and a s-p cross-term (which we can denote as SP-TAM). A lot can be learned on the physics governing the TAM by expressing any field in terms of vectorial Helmholtz Natural Modes (HNMs) $[16,17]$. As we will show in this paper, this allows us to write the angular momentum per unit energy as made of three parts ( $n$ denotes the azimuthal charge carried by the field and $m$ the radial one)

$$
\begin{aligned}
\gamma & =\frac{\sigma_{z}}{\omega}+\frac{1}{\omega} \frac{\operatorname{Re}\left\{\sum_{m, n} \frac{4}{3} n\left|c_{m, n}\right|^{2}+\sum_{\substack{m, m^{\prime}, n \\
m \neq m^{\prime}}} n c_{m^{\prime}, n}^{\star} c_{m, n} f\left(m, m^{\prime}\right)\right\}}{\sum_{m, n} \frac{4}{3}\left|c_{m, n}\right|^{2}+\sum_{\substack{m, m^{\prime}, n \\
m \neq m^{\prime}}} c_{m^{\prime}, n}^{\star} c_{m, n} f\left(m, m^{\prime}\right)}+ \\
& +\frac{\sigma_{z}}{\omega} \frac{\operatorname{Re}\left\{\sum_{m, n} \frac{2}{3}\left|c_{m, n}\right|^{2}-\sum_{\substack{m, m^{\prime}, n \\
m \neq m^{\prime}}}^{\star} c_{m^{\prime}, n}^{\star} c_{m, n} f\left(m, m^{\prime}\right)\right\}}{\sum_{m, n} \frac{4}{3}\left|c_{m, n}\right|^{2}+\sum_{\substack{m, m^{\prime}, n \\
m \neq m^{\prime}}} c_{m^{\prime}, n}^{\star} c_{m, n} f\left(m, m^{\prime}\right)}
\end{aligned}
$$

Eq. 3 tells us that the TAM has three main contributions: a first term only dependent on the spin $\sigma_{z}$, a second one dependent on the azimuthal charges $n$ present in the field (the orbital part) and a last term which depends on the spin $\sigma_{z}$ and the radial charges $m$ contained in the field. $\mathfrak{J}_{z} / W$ can be interpreted as if each photon in the field contributes to the angular momentum through a term $\sigma_{z} \hbar$ due to its spin, a term $n \hbar$ due to its orbital angular momentum and a third term, again proportional to the spin $\sigma_{z}$ and function of the radial charges carried by the field. The last contribution, which is absent within the paraxial regime, is a special trait of the electromagnetic theory. In the talk we will discuss on the coupling among radial charges, identify cases when such coupling is absent and the interesting case where no azimuthal charge is present. [18].

\section{References}

[1] L. Allen, M.W. Beijersbergen, R.J.C., Spreeuw and J.P. Woerdman, “Orbital angular momentum of light and the transformation of Laguerre-Gaussian laser modes" Phys. Rev. A, 45, 8185, 1992. 


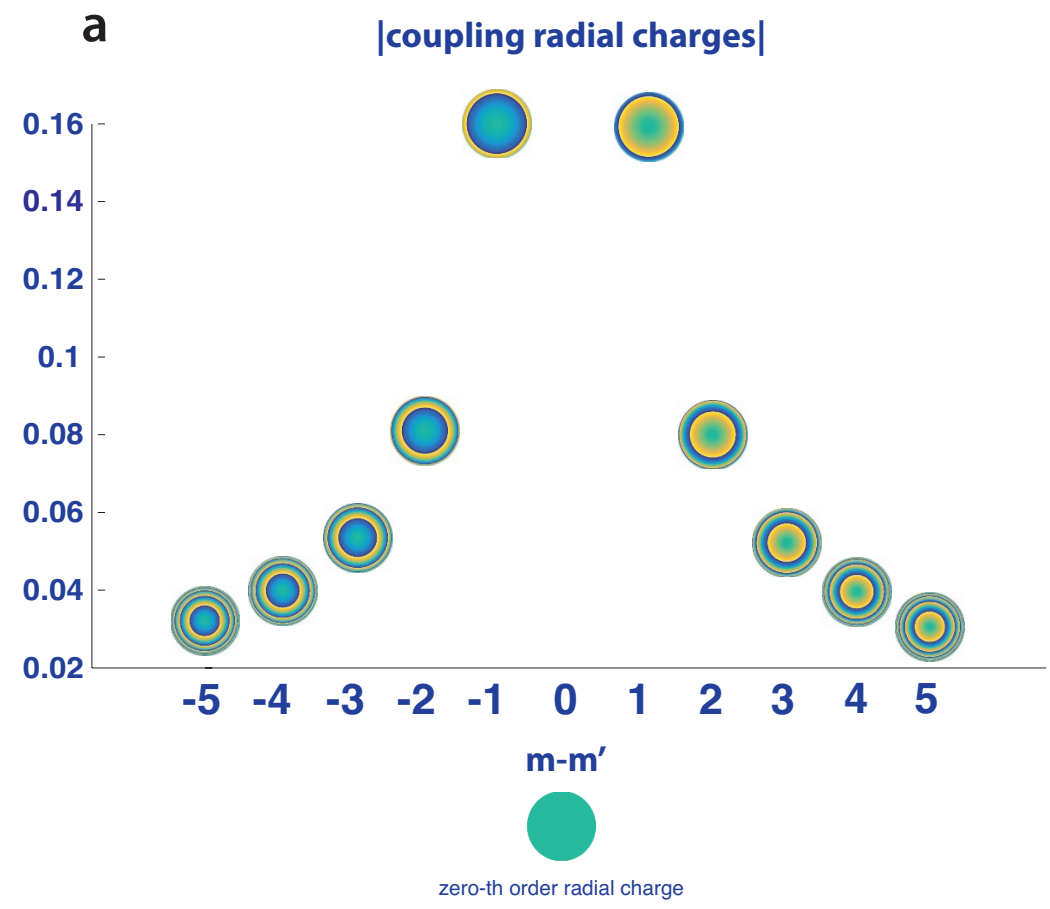

Figure 1. Panel a: Absolute value of the coupling function $f\left(m, m^{\prime}\right)=1 /\left[2 i \pi\left(m-m^{\prime}\right)\right]+1 /\left[2 \pi^{2}\left(m-m^{\prime}\right)^{2}\right]$ among neighbouring radial modes. The curve refers to the coupling of the 0 -th order radial charge (shown close to the origin of the $\mathrm{x}$-axis in the graph) with the neighbouring radial charges, of order $m \in[-5,5]$. On the $\mathrm{x}$-axis the order of each charge is reported, while the phase profile of each charge is used as marker on the curve. Only radial charges corresponding to HNMs of same azimuthal charge $n$ can couple to each other.

[2] Quantum Imaging, M. Kolobov Editor, Springer Singapore, 2007.

[3] Quantum metrology, Imaging and Communication, D.S. Simon, G. Jaeger, A.V. Sergienko Editors, Springer Cham, Switzerland, 2017.

[4] Y. Yan, G. Xie, M. P. J. Lavery, H. Huang, N. Ahmed, C. Bao, Y. Ren, Y. Cao, L. Li, Z. Zhao, A. F. Molisch, M. Tur, M. J. Padgett, A. E. Willner, "High-capacity millimetre-wave communications with orbital angular momentum multiplexing", Nature Comm., 5, 4876, 2014.

[5] T. Van Mechelen and Z. Jacob, "Universal spin-momentum locking of evanescent waves", Optica, 3, 118 - 126, 2016.

[6] L. Allen, M.J.Padgett and M. Babiker, "The orbital angular momentum of light", in Progress in Optics XXXIX, Elsevier, Amsterdam, Netherlands, E. Wolf Editor, 291-372, 1999.

[7] C. Cohen-Tannoudji, J. Dupont-Roc, G. Grynberg, Photons \& Atoms, Wiley-VHC Weinheim, Germany, 2004.

[8] S. M. Barnett, L. Allen, "Orbital angular momentum and non paraxial light beams", Optics Comm., 110, 670-678, 1994.

[9] K. Y. Bliokh, M. A. Alonso, E. A. Ostrovskaya, and A. Aiello, "Angular momenta and spin-orbit interaction of nonparaxial light in free space", PRA, 82, 063825, 2010. 
[10] S.J. van Enk, G. Nienhuis, "Commutation rules and eigenvalues of spin and orbital angular momentum of radiation fields”, J. Modern Opt. 41, 963-977, 1994.

[11] K. Y. Bliokh, F. Nori, "Transverse and longitudinal angular momenta of light", Physics Reports, 592, 1-38, (2015).

[12] M. S. Soskin, V. N. Gorshkov, M. V. Vasnetsov, J. T. Malos, and N. R. Heckenberg, "Topological charge and angular momentum of light beams carrying optical vortices", Phys. Rev. A 56, 4064, 1997.

[13] S. M. Barnett, L. Allen, R. P. Cameron, C. R. Gilson, M. J. Padgett, F. C. Speirits and A. M. Yao, "On the natures of the spin and orbital parts of optical angular momentum", Journal of Optics, 18, 064004, 2016.

[14] S. M. Barnett, M. Babiker, M. J. Padgett, "Optical orbital angular momentum", Phil. Trans. R. Soc. A 375, 20150444, 2017.

[15] K. Y. Bliokh, F. J. Rodriguez-Fortuno, F. Nori, A. Zayats, "Spin-orbit interactions of light”, Nature Phot.,9, 796-808, 2015.

[16] O. El Gawhary, "Helmholtz Natural Modes: the universal and discrete spatial fabric of electromagnetic wavefields ", New J. Phys. 19, 013021, 2017.

[17] O. El Gawhary, "On a propagation-invariant, orthogonal modal expansion on the unit disk: going beyond Nijboer-Zernike theory of aberrations", Opt. Lett. 40, 2626-2629, 2015.

[18] O. El Gawhary, T. van Mechelen, H.P. Urbach, "Role of Radial Charges on the Angular Momentum of Electromagnetic Fields: Spin-3/2 Light”, Phys. Rev. Lett., 121, 123202, 2018. 